\title{
New Mersenne Primes
}

\section{By Alexander Hurwitz}

If $p$ is prime, $M_{p}=2^{p}-1$ is called a Mersenne number. The primes $M_{4253}$ and $M_{4423}$ were discovered by coding the Lucas-Lehmer test for the IBM 7090. These two new primes are the largest prime numbers known; for other large primes see Robinson [4]. The computing was done at the UCLA Computing Facility. This test is described by the following theorem (see Lehmer [1, p. 443-4]).

TheOREM. If $S_{1}=4$ and $S_{n+1}={S_{n}}^{2}-2$ then $M_{p}$ is prime if and only if $S_{p-1} \equiv 0$ $\left(\bmod M_{p}\right)$.

The test takes about 50 minutes of machine time for $p=4423$. These results bring the number of known Merserine primes to 20. The values of $p$ for these twenty primes are listed in Table 1.

If $M_{p}$ is prime it is of interest to know the sign of the least absolute penultimate residue, that is, whether $S_{p-2} \equiv+2^{r}\left(\bmod M_{p}\right)$ or $S_{p-2} \equiv-2^{r}\left(\bmod M_{p}\right)$ where $2 r=p+1$. The Lucas-Lehmer test can also be used with $S_{1}=10$. The various penultimate residues of the known Mersenne primes were computed and the results appear in Table 1 (see Robinson [3]).

In addition to testing the above Mersenne primes each Mersenne number with $p<5000$ was tested unless a factor of $M_{p}$ was known. The residues of $S_{p-1}(\bmod$ $\left.M_{p}\right)$ are available for checking purposes. The results for $3300<p<5000$ are summarized in Table 2. The computer program also found (see [3, p. 844]) that $M_{8191}$ is not prime.

The residue $S_{p-1}\left(\bmod M_{p}\right)$ for $p>3300$ is output from the computer in a modified octal notation. That is, the residue is stored in the computer in 35 bit binary words and the output is a word by word conversion of the 35 bit words into octal (base 8) numbers. Thus the leading digit of each is quaternary (base 4). For $p<3300$ the residue was printed in hexadecimal notation (see Robinson [3] and Riesel [2]).

\section{TABLE 1}

\begin{tabular}{c|c|c||c|c|c}
\hline$p$ & $S_{1}=4$ & $S_{1}=10$ & $p$ & $S_{1}=4$ & $S_{1}=10$ \\
\cline { 2 - 5 } & & & 107 & - & + \\
3 & + & - & 127 & + & + \\
5 & + & - & 521 & - & + \\
7 & - & - & 607 & - & - \\
13 & + & + & 1279 & - & - \\
17 & - & + & 2203 & + & - \\
19 & - & + & 2281 & - & + \\
31 & + & + & 3217 & - & + \\
61 & + & + & 4253 & + & + \\
89 & - & + & 4423 & - & -
\end{tabular}

Received November 3, 1961. The preparation of this paper was sponsored by the U. S. Office of Naval Research. 
The five least significant octal digits of the residue appear in Table 2 for each $p>3300$ tested. If $p(3300<p<5000)$ is omitted from Table 2 a factor of $2^{p}-1$ is known. Some of these factors are not yet published but were communicated to the author by John Brillhart.

My thanks to the Computing Facility for their help in this work, especially J. L. Selfridge and F. H. Hollander.

University of California at Los Angeles

Los Angeles, California

1. D. H. LeHMER, "An extended theory of Lucas' functions," Ann. of Math. v. 31, 1930, p. $419-448$.

2. H. Riesel, "Mersenne numbers," MTAC, v. 12, 1958, p. 207-213.

3. R. M. Robinson, "Mersenne and Fermat numbers," Proc. Amer. Math. Soc. v. 5, 1954, p. 842-846.

4. R. M. Robinson, "A report on primes of the form $k \cdot 2^{n}+1$ and on factors of Fermat numbers," Proc. Amer. Math. Soc. v. 9, 1958, p. 673-681. 